http:/www.journals.zu.edu.eg/journalDisplay.aspx?Journalld=1\&queryType=Master

\title{
IMPACT OF HUMIC ACID APPLICATION AND NITROGEN FERTILIZER LEVEL ON BREAD WHEAT PRODUCTIVITY UNDER SANDY SOIL CONDITIONS
}

\author{
Mohamed A.T. Yasin " and E.E.A. El-Sobky \\ Agron. Dept., Fac. Agric., Zagazig Univ., Egypt
}

Received: 28/03/2017 ; Accepted: 02/04/2017

\begin{abstract}
Two field experiments were carried out at the Agricultural Research Station, Faculty of Agriculture, Zagazig University, El-Khattara region, Sharkia Governorate, Egypt during the two successive seasons of 2014/2015 and 2015/2016 to investigate the effect of three rates of humic acid application (0, 2 and $4 \mathrm{~kg}$ humic acid/fad.) and five nitrogen fertilizer levels $(0,30,60,90$ and $120 \mathrm{~kg}$ $\mathrm{N} /$ fad.) on bread wheat (Triticum aestivum L. cv. Sids 12) grown in sandy soil under sprinkler irrigation system. Results of the combined analysis indicated that application of humic acid at $2 \mathrm{~kg} /$ fad., significantly increased plant height $(\mathrm{cm})$, flag leaf area $\left(\mathrm{cm}^{2}\right)$, spike length $(\mathrm{cm})$, number of spikes $/ \mathrm{m}^{2}$, straw yield (ton/fad.) and harvest index as compared to control treatment. Furthermore, flag leaf chlorophyll content (SPAD), number of grains/spike, weight of grains/spike (g), 1000-grain weight (g), grain and biological yields (ton/fad.) showed significant increase due to raising humic acid application up to $4 \mathrm{~kg} /$ fad. On the other hand, it was found that plant height, spike length, number and weight of grains/spike, number of spikes $/ \mathrm{m}^{2}, 1000$-grain weight, straw yield and harvest index were significantly increased by increasing nitrogen fertilizer levels up to $90 \mathrm{~kg} \mathrm{~N} / \mathrm{fad}$. Whereas, flag leaf area, flag leaf chlorophyll content, grain and biological yields per fad responded to each $\mathrm{N}$ increment up to $120 \mathrm{~kg} \mathrm{~N} /$ fad. Most studied traits were significantly affected by the interaction between humic acid and nitrogen fertilizer levels. It could be noticed that, application of humic acid resulted in reducing the requirement to $\mathrm{N}$ fertilizer application. The highest grain yield (16.25 ardab/fad.) was achieved by soil application of $4 \mathrm{~kg}$ humic acid with $90 \mathrm{~kg} \mathrm{~N} / \mathrm{fad}$.
\end{abstract}

Key words: Wheat, nitrogen, humic, chlorophyll content.

\section{INTRODUCTION}

Wheat (Triticum aestivum, L.) is dominant grain crop all over the world as well as in Egypt. It is occupying an important part of the daily diet of millions people. The total cultivated area of wheat in Egypt reached about 1.43 million hectares and the total production overtake 9.28 million tons with an average of 6.51 tons/ha (FAOSTAT, 2016). Using organic fertilizers is very important in food safety and environmental health, therefore it is taken into consideration all over the world. The stimulatory effects of humic substances were directly linked to the improved nutrients uptake (Chen and Aviad, 1990; Chen et al., 1999). Furthermore, the effects of humic acid on soil fertility via increased microbial population of microorganisms, as well as, improved chemical and physical properties of soil. Moreover, humic acid may had varying biochemical effects on the cell wall, membrane level and the cytoplasm which had caused an increase in photosynthesis and respiration, enhanced protein synthesis and plant hormones activities (Chen and Aviad, 1990). Among organic fertilizers, humic acid considers as a principal component of humic substances, which are the main components of soil. When appropriate humic substances are exist within the soil, nitrogen, phosphorus and potassium

\footnotetext{
*Corresponding author: Tel. : +201000843169

E-mail address: taha_agronomy_1978@yhaoo.com
} 
fertilizer application rates may be reduced (Pettit, 2004). Humic acid had many beneficial effects on soil properties which promotes plant growth and nutrient uptake, as well as increasing yield (Cimrin and Yilmaz, 2005; Asik et al., 2009). Khaled and Fawy (2011) reported that humic acid can reduce water loss through evaporation and increase water use efficiency by plants due to increased the water holding capacity of sandy soils. Humic acid was also found to plant chlorosis, increase the permeability of the plant membranes, intensify enzyme systems of plants, accelerate cell division, show greater root development, and decrease stress deterioration. Humic acid reduce soil erosion via increasing the cohesive forces of the fine soil particles, promoting of elements chelation and hence, this might play an important role in enriching soil fertility and availability of nutrients. In this respect, many investigators reported significant increase in yield and yield components of wheat due to the application of humic acid (Antoun et al., 2010; Khan et al., 2010; El-Hamdi et al., 2012; Bakry et al., 2013; Tufail et al., 2014; Radwan et al., 2015; Anwar et al., 2016). Moreover, Kandil et al. (2016) and Manal et al. (2016) showed that higher averages of spike length, number of grains/spike, grain weight/spike, 1000-grain weight as well as grain, straw and biological yields of wheat were obtained by application of humic acid, while harvest index was significantly decreased.

Egyptian soils, especially sandy soils, have low residual soil nitrogen content as a result of low content of organic matter and the small amount of organic manures added to it annually. Therefore, nitrogen fertilization is considering one of the key factors for increasing wheat yield. Moussa and Abdel-Maksoud (2011) showed that, increasing nitrogen fertilizer level up to 90 $\mathrm{Kg} \mathrm{N} / \mathrm{fad}$., tended to cause significant increase in wheat grain yield and yield components i.e. spike number $/ \mathrm{m}^{2}$, grain number/spike and 1000 grain weight. Other researchers recorded significant increment in wheat grain and straw yields as well as yield components by raising nitrogen fertilizer level up to $100 \mathrm{Kg} \mathrm{N} /$ fad., (Zeidan et al., 2005; Antoun et al., 2010; Zaki et al., 2012; Khaled and Hammad, 2014). Furthermore, Ali et al. (2004) and Mowafy
(2008) apprehended that nitrogen fertilizer addition up to $120 \mathrm{~kg} \mathrm{~N} /$ fad., caused significant increase in plant height, spike number $/ \mathrm{m}^{2}$, grain weight/spike, 1000- grain weight, grain and straw yields/fad. Farag and El-Khawaga (2013) manifested that each increment in nitrogen level from 25 to $125 \mathrm{~kg} \mathrm{~N} /$ fad., caused significant increases in yield and yield attributes of wheat. Recently, Kandil et al. (2016) revealed that wheat yield attributes, grain and straw yields per ha were significantly increased due to raising nitrogen fertilizer level from 166, 214 to $262 \mathrm{Kg}$ $\mathrm{N} / \mathrm{ha}$.

Therefore, this investigation was conducted to study the effect of soil application of humic acid and nitrogen fertilizer levels on yield and yield attributes of bread wheat (Sids 12 cultivar) grown in newly reclaimed sandy soil under sprinkler irrigation system.

\section{MATERIALS AND METHODS}

\section{Study Site and Objective}

Two field experiments were conducted out at the Agricultural Research Station, Faculty of Agriculture, Zagazig University at El-Khattara region, Sharkia Governorate, Egypt during the two successive seasons of 2014/2015 and 2015/ 2016 to study the effect of humic acid rates and nitrogen fertilizer levels on yield and its attributes of bread wheat (Sids 12 cultivar) grown in sandy soil under sprinkler irrigation system.

\section{Experimental Design and Treatments}

The experiment was laid out in randomized complete block design using split plot arrangement of treatments with four replicates. The main plots were allocated for three soil application rates of humic acid i.e. 0, 2 and 4 $\mathrm{Kg}$ /fad., where humic acid was soil incorporated before sowing. The sub-plots were assigned for five nitrogen fertilizer levels i.e. $0,30,60,90$ and $120 \mathrm{Kg} \mathrm{N} / \mathrm{fad}$., as ammonium sulphate $(20.5 \% \mathrm{~N})$ which was applied in five equal doses, the first was after 15 days from sowing, and then every 15 days. The sub plot area was $14 \mathrm{~m}^{2}$ (3.5 width $\times 4 \mathrm{~m}$ length) included 20 rows each of $4 \mathrm{~m}$ length and $17.5 \mathrm{~cm}$ apart. The soil of the experimental site was sandy in texture. Soil samples were collected to depth of $30 \mathrm{~cm}$ 
from the experimental site before planting to determine soil physical and chemical properties (Central Laboratory of Faculty of Agriculture, Zagazig University, Zagazig, Egypt). The experimental soil was sandy in texture where it has a particle size distribution of $92.98,5.75$ and $1.27 \%$ for sand, silt and clay, respectively. The soil had an average $\mathrm{pH}$ of 8.15 and organic matter content of $0.42 \%$. The average available $\mathrm{N}, \mathrm{P}$ and $\mathrm{K}$ contents were 15.0, 6.1 and 76.0 ppm, respectively.

\section{Agricultural Practices}

The preceding crop was maize (Zea mays, L.) in both seasons of the study. The grains of Sids 12 wheat cultivar were sown at a seeding rate of $75 \mathrm{Kg} / \mathrm{fad}$., on $24^{\text {th }}$ and $23^{\text {rd }}$ November in the first and the second seasons, respectively. Phosphorus fertilizer was added during seed bed preparation at rate of $31 \mathrm{Kg} \mathrm{P}_{2} \mathrm{O}_{5} /$ fad., in the form of calcium superphosphate $\left(15.5 \% \mathrm{P}_{2} \mathrm{O}_{5}\right)$, while potassium fertilizer was applied at rate of $48 \mathrm{~kg} \mathrm{~K} \mathrm{~K}_{2} \mathrm{O} / \mathrm{fad}$., as potassium sulphate $(48 \%$ $\mathrm{K}_{2} \mathrm{O}$ ) in one dose with the first dose of nitrogen fertilizer. Wheat plants were grown under sprinkler irrigation system, solid system (130 $\mathrm{m}^{3} / \mathrm{hr}$.) where irrigation was followed twice weekly. The harvest was performed on $1^{\text {st }}$ may in both seasons.

\section{Studied Characters}

At heading, ten guarded plants were taken at random from each experimental plot to determine plant height $(\mathrm{cm})$, flag leaf area $\left(\mathrm{cm}^{2}\right)$ which was determined according to Lui and Subbo Roo (1951), In addition to flag leaf chlorophyll content, which was measured as SPAD units with handheld chlorophyll meter (SPAD-502 plus), according to Castelli et al. (1996). At harvest, ten spikes were taken at random from each experimental plot to estimate the following traits: spike length $(\mathrm{cm})$, number of grains/spike and weight of grains/spike (g). Thereafter, bulk sample included all wheat plants in $2 \mathrm{~m}^{2}$ in each sub plot was used to measure number of spikes $/ \mathrm{m}^{2}, 1000$-grain weight (g), grain yield (ardab/fad.), straw yield (ton/fad.), biological yield (ton/fad.) and harvest index $(\mathrm{HI})$.

\section{Statistical Analysis}

The obtained data of the two seasons and their combined were statistically analyzed as mentioned by Gomez and Gomez (1991) using the computer MSTAT statistical analysis package (MSTAT-C, 1991). Least significant differences (LSD) method was used to test the differences between treatment means at 5\% level of probability as described by Steel et al. (1997). The error mean squares of split plot design were homogenous (Bartlett's test), the combined analysis was calculated for all the studied characters in both seasons. In interaction Tables, capital letters were used to compare the values in rows, while small letters were used to compare the values in columns.

\section{RESULTS AND DISCUSSION}

\section{Effect of Humic Acid Rates}

\section{Yield attributes}

Results presented in Tables 1, 2 and 3 indicate that humic acid application had a marked effect on all studied yield attributes traits in both seasons and their combined analysis. Application of humic acid at $2 \mathrm{Kg} / \mathrm{fad}$., significantly increased each of plant height, flag leaf area, spike length and number of spikes $/ \mathrm{m}^{2}$, as compared to control treatment, concerning to the combined analysis. Moreover, flag leaf chlorophyll content, number and weight of grains/spike as well as 1000-grain weight exhibited significant increase due to raising humic acid application up to $4 \mathrm{Kg} / \mathrm{fad}$, regarding to the combined results. The beneficial effects of humic acid on plant growth may be ascribed to the increasing of cell membrane, oxygen uptake, respiration and photosynthesis, nutrients uptake, root and cell elongation and ion transport (Nardi et al., 2002).

\section{Grain, straw, biological yields and harvest index}

Results given in Tables 3 and 4 show significant increments in grain, straw and biological yields as well as harvest index during both growing seasons and their combined analysis due to soil application of humic acid, as compared to without humic acid application. Regarding to the combined analysis results, it could be mentioned that soil application of humic acid at $2 \mathrm{Kg} / \mathrm{fad}$., significantly increased straw yield and harvest index. Furthermore, grain and biological yields showed significant 
Table 1. Plant height $(\mathrm{cm})$, flag leaf area $\left(\mathrm{cm}^{2}\right)$ and chlorophyll content (SPAD value) of wheat as affected by humic acid rate, nitrogen fertilizer level and their interaction during both seasons and their combined analysis

\begin{tabular}{|c|c|c|c|c|c|c|c|c|c|}
\hline \multirow[t]{2}{*}{ Main effects and interaction } & \multicolumn{3}{|c|}{$\begin{array}{l}\text { Plant height } \\
\text { (cm) }\end{array}$} & \multicolumn{3}{|c|}{$\begin{array}{l}\text { Flag leaf area } \\
\left(\mathrm{cm}^{2}\right)\end{array}$} & \multicolumn{3}{|c|}{$\begin{array}{c}\text { Chlorophyll content } \\
\text { (SPAD value) }\end{array}$} \\
\hline & $\begin{array}{c}1^{\text {st }} \\
\text { season } \\
\end{array}$ & $\begin{array}{c}2^{\text {nd }} \\
\text { season } \\
\end{array}$ & $\overline{\text { Comb. }}$ & $\begin{array}{c}1^{\text {st }} \\
\text { season } \\
\end{array}$ & $\begin{array}{c}2^{\text {nd }} \\
\text { season } \\
\end{array}$ & $\overline{\text { Comb. }}$ & $\begin{array}{c}1^{\text {st }} \\
\text { season } \\
\end{array}$ & $\begin{array}{c}2^{\text {nd }} \\
\text { season } \\
\end{array}$ & Comb. \\
\hline \multicolumn{10}{|l|}{ Humic acid rate $(\mathrm{H})$} \\
\hline 0 kg/fad. & $77.87 \mathrm{~b}$ & $76.67 b$ & $77.27 b$ & $24.19 b$ & $20.99 b$ & $22.59 b$ & $37.26 \mathrm{~b}$ & $34.99 b$ & $36.13 \mathrm{c}$ \\
\hline 2 kg/fad. & $85.53 \mathrm{a}$ & $78.87 \mathrm{~b}$ & $82.20 \mathrm{a}$ & $27.48 \mathrm{a}$ & $23.76 \mathrm{a}$ & $25.62 \mathrm{a}$ & $42.66 \mathrm{a}$ & $39.94 \mathrm{ab}$ & $41.30 \mathrm{~b}$ \\
\hline 4 kg/fad. & $83.47 \mathrm{a}$ & $85.60 \mathrm{a}$ & $84.54 \mathrm{a}$ & $29.01 \mathrm{a}$ & $25.57 \mathrm{a}$ & $27.29 \mathrm{a}$ & $46.76 \mathrm{a}$ & $44.37 \mathrm{a}$ & $45.57 \mathrm{a}$ \\
\hline F-test & $*$ & $*$ & $* *$ & $* *$ & $* *$ & $* *$ & $*$ & $*$ & $* *$ \\
\hline \multicolumn{10}{|l|}{ Nitrogen fertilizer level (N) } \\
\hline 0 kg N/fad. & $61.78 \mathrm{~d}$ & $67.11 d$ & $64.45 \mathrm{~d}$ & $18.87 \mathrm{e}$ & $15.49 \mathrm{e}$ & $17.18 \mathrm{e}$ & $36.43 d$ & $34.02 \mathrm{~d}$ & $35.23 \mathrm{e}$ \\
\hline $30 \mathrm{~kg} \mathrm{~N} /$ fad. & $73.6 \mathrm{zc}$ & $74.11 \mathrm{c}$ & $73.89 \mathrm{c}$ & $23.12 \mathrm{~d}$ & $19.70 \mathrm{~d}$ & $21.41 d$ & $39.38 \mathrm{c}$ & $36.72 \mathrm{c}$ & $38.05 d$ \\
\hline $60 \mathrm{~kg} \mathrm{~N} /$ fad. & $85.11 \mathrm{~b}$ & $81.78 b$ & $83.45 b$ & $27.40 \mathrm{c}$ & $23.93 \mathrm{c}$ & $25.67 \mathrm{c}$ & $42.98 b$ & $40.39 \mathrm{~b}$ & $41.69 \mathrm{c}$ \\
\hline $90 \mathrm{~kg} \mathrm{N/fad.}$ & $94.33 \mathrm{a}$ & $87.89 a$ & $91.11 \mathrm{a}$ & $31.21 \mathrm{~b}$ & $27.67 b$ & $29.44 b$ & $44.90 \mathrm{~b}$ & $42.47 b$ & $43.69 b$ \\
\hline $120 \mathrm{~kg} \mathrm{~N} /$ fad. & $96.56 a$ & $91.00 \mathrm{a}$ & $93.78 \mathrm{a}$ & $33.87 \mathrm{a}$ & $30.40 \mathrm{a}$ & $32.14 \mathrm{a}$ & $47.44 \mathrm{a}$ & $45.23 \mathrm{a}$ & $46.34 \mathrm{a}$ \\
\hline F-test & $* *$ & $* *$ & $* *$ & $* *$ & $* *$ & $* *$ & $* *$ & $* *$ & $* *$ \\
\hline \multicolumn{10}{|l|}{ interaction } \\
\hline $\mathbf{H} \times \mathbf{N}$ & $*$ & $*$ & $*$ & $*$ & $*$ & $* *$ & NS & NS & $* *$ \\
\hline
\end{tabular}

$*, * *$ and NS indicate significant at $0.05,0.01$ and not significant, respectively.

Table 2. Spike length (cm), number of grains/spike and weight of grains/spike (g) of wheat as affected by humic acid rate, nitrogen fertilizer level and their interaction during both seasons and their combined analysis

\begin{tabular}{|c|c|c|c|c|c|c|c|c|c|}
\hline \multirow[t]{2}{*}{ Main effects and interaction } & \multicolumn{3}{|c|}{$\begin{array}{l}\text { Spike length } \\
\text { (cm) }\end{array}$} & \multicolumn{3}{|c|}{$\begin{array}{c}\text { No. of } \\
\text { grains/spike }\end{array}$} & \multicolumn{3}{|c|}{$\begin{array}{c}\text { Weight of } \\
\text { grains/spike (g) }\end{array}$} \\
\hline & $\begin{array}{c}1^{\text {st }} \\
\text { season }\end{array}$ & $\begin{array}{c}2^{\text {nd }} \\
\text { season }\end{array}$ & $\overline{\text { Comb. }}$ & $\begin{array}{c}1^{\text {st }} \\
\text { season }\end{array}$ & $\begin{array}{c}2^{\text {nd }} \\
\text { season }\end{array}$ & $\overline{\text { Comb. }}$ & $\begin{array}{c}1^{\text {st }} \\
\text { season }\end{array}$ & $\begin{array}{c}2^{\text {nd }} \\
\text { season }\end{array}$ & Comb. \\
\hline \multicolumn{10}{|l|}{ Humic acid rate (H) } \\
\hline 0 kg/fad. & $9.09 \mathrm{~b}$ & $9.59 \mathrm{~b}$ & $9.34 \mathrm{~b}$ & $34.73 \mathrm{~b}$ & $33.27 \mathrm{~b}$ & $34.00 \mathrm{c}$ & $1.162 \mathrm{c}$ & $1.111 \mathrm{~b}$ & $1.137 \mathrm{c}$ \\
\hline 2 kg/fad. & $9.67 \mathrm{ab}$ & $10.64 \mathrm{a}$ & $10.16 \mathrm{a}$ & $38.33 \mathrm{a}$ & $37.20 \mathrm{a}$ & $37.77 \mathrm{~b}$ & $1.431 \mathrm{~b}$ & $1.476 \mathrm{a}$ & $1.454 \mathrm{~b}$ \\
\hline 4 kg/fad. & $10.05 \mathrm{a}$ & $11.03 \mathrm{a}$ & $10.54 \mathrm{a}$ & $41.00 \mathrm{a}$ & $39.07 \mathrm{a}$ & $40.04 \mathrm{a}$ & $1.650 \mathrm{a}$ & $1.489 \mathrm{a}$ & $1.570 \mathrm{a}$ \\
\hline F-test & $*$ & $* *$ & $* *$ & $*$ & $*$ & $* *$ & $* *$ & $* *$ & $* *$ \\
\hline \multicolumn{10}{|l|}{ Nitrogen fertilizer level (N) } \\
\hline 0 kg N/fad. & $7.54 \mathrm{c}$ & $9.52 \mathrm{~d}$ & $8.53 \mathrm{c}$ & $30.44 \mathrm{~d}$ & $29.67 \mathrm{~d}$ & $30.06 \mathrm{~d}$ & $0.954 \mathrm{~d}$ & $0.874 \mathrm{~d}$ & $0.914 \mathrm{~d}$ \\
\hline 30 kg N/fad. & $9.49 \mathrm{~b}$ & $10.19 \mathrm{c}$ & $9.84 \mathrm{~b}$ & $33.44 \mathrm{c}$ & $33.11 \mathrm{c}$ & $33.28 \mathrm{c}$ & $1.197 \mathrm{c}$ & $1.136 \mathrm{c}$ & $1.167 \mathrm{c}$ \\
\hline $60 \mathrm{~kg} \mathrm{~N} / \mathrm{fad}$ & $9.82 \mathrm{~b}$ & $10.45 b c$ & $10.14 \mathrm{~b}$ & $38.22 \mathrm{~b}$ & $36.44 \mathrm{~b}$ & $37.33 \mathrm{~b}$ & $1.453 \mathrm{~b}$ & $1.352 \mathrm{~b}$ & $1.403 \mathrm{~b}$ \\
\hline $90 \mathrm{~kg}$ N/fad. & $10.51 \mathrm{a}$ & $10.88 \mathrm{ab}$ & $10.70 \mathrm{a}$ & $43.00 \mathrm{a}$ & $40.78 \mathrm{a}$ & $41.89 \mathrm{a}$ & $1.702 \mathrm{a}$ & $1.662 \mathrm{a}$ & $1.682 \mathrm{a}$ \\
\hline $120 \mathrm{~kg} \mathrm{~N} /$ fad. & $10.66 \mathrm{a}$ & $11.05 \mathrm{a}$ & $10.86 \mathrm{a}$ & $45.00 \mathrm{a}$ & $42.56 \mathrm{a}$ & $43.78 \mathrm{a}$ & $1.764 \mathrm{a}$ & $1.770 \mathrm{a}$ & $1.767 \mathrm{a}$ \\
\hline F-test & $* *$ & $* *$ & $* *$ & $* *$ & $* *$ & $* *$ & $* *$ & $* *$ & $* *$ \\
\hline \multicolumn{10}{|l|}{ interaction } \\
\hline $\mathbf{H} \times \mathbf{N}$ & NS & NS & NS & NS & NS & NS & $* *$ & NS & $* *$ \\
\hline
\end{tabular}

$*, * *$ and NS indicate significant at $0.05,0.01$ and not significant, respectively. 
Table 3. Number of spikes $/ \mathrm{m}^{2}, 1000$-grain weight (g) and grain yield (ardab/fad.) of wheat as affected by humic acid rate, nitrogen fertilizer level and their interaction during both seasons and their combined analysis

\begin{tabular}{|c|c|c|c|c|c|c|c|c|c|}
\hline \multirow[t]{2}{*}{ Main effects and interaction } & \multicolumn{3}{|c|}{$\begin{array}{c}\text { No. of } \\
\text { spikes } / \mathbf{m}^{2}\end{array}$} & \multicolumn{3}{|c|}{$\begin{array}{l}\text { 1000-grain } \\
\text { weight (g) }\end{array}$} & \multicolumn{3}{|c|}{$\begin{array}{l}\text { Grain yield } \\
\text { (ardab/fad.) }\end{array}$} \\
\hline & $\begin{array}{c}1^{\text {st }} \\
\text { season }\end{array}$ & $\begin{array}{c}2^{\text {nd }} \\
\text { season }\end{array}$ & $\overline{\text { Comb. }}$ & $\begin{array}{c}1^{\text {st }} \\
\text { season }\end{array}$ & $\begin{array}{c}2^{\text {nd }} \\
\text { season }\end{array}$ & Comb. & $\begin{array}{c}1^{\text {st }} \\
\text { season }\end{array}$ & $\begin{array}{c}2^{\text {nd }} \\
\text { season }\end{array}$ & Comb. \\
\hline \multicolumn{10}{|l|}{ Humic acid rate $(\mathrm{H})$} \\
\hline 0 kg/fad. & $276.1 \mathrm{~b}$ & $289.3 \mathrm{~b}$ & $282.7 \mathrm{~b}$ & $35.05 \mathrm{~b}$ & $36.11 \mathrm{~b}$ & $35.58 \mathrm{c}$ & $9.68 \mathrm{c}$ & $9.06 \mathrm{c}$ & $9.37 \mathrm{c}$ \\
\hline 2 kg/fad. & $330.5 \mathrm{a}$ & $348.1 \mathrm{a}$ & $339.3 \mathrm{a}$ & $39.58 \mathrm{a}$ & $38.55 \mathrm{ab}$ & $39.07 \mathrm{~b}$ & $11.53 \mathrm{~b}$ & $11.19 b$ & $11.36 \mathrm{~b}$ \\
\hline 4 kg/fad. & $341.4 \mathrm{a}$ & $361.2 \mathrm{a}$ & $351.3 \mathrm{a}$ & $42.77 \mathrm{a}$ & $40.63 \mathrm{a}$ & $41.70 \mathrm{a}$ & $12.84 \mathrm{a}$ & $12.78 \mathrm{a}$ & $12.81 \mathrm{a}$ \\
\hline F-test & $* *$ & * & $* *$ & $*$ & $*$ & $* *$ & $* *$ & $* *$ & $* *$ \\
\hline \multicolumn{10}{|l|}{ Nitrogen fertilizer level (N) } \\
\hline 0 kg N/fad. & $254.9 \mathrm{~d}$ & $275.1 \mathrm{~d}$ & $265.0 \mathrm{~d}$ & $33.24 \mathrm{~d}$ & $31.17 \mathrm{~d}$ & $32.21 \mathrm{~d}$ & $6.79 \mathrm{~d}$ & $5.61 \mathrm{e}$ & $6.21 \mathrm{e}$ \\
\hline 30 kg N/fad. & $285.7 \mathrm{c}$ & $305.9 \mathrm{c}$ & $295.8 \mathrm{c}$ & $36.88 \mathrm{c}$ & $35.93 \mathrm{c}$ & $36.41 \mathrm{c}$ & $8.85 \mathrm{c}$ & $8.32 \mathrm{~d}$ & $8.59 \mathrm{~d}$ \\
\hline $60 \mathrm{~kg} \mathrm{~N} / \mathrm{fad}$ & $320.8 \mathrm{~b}$ & $335.3 \mathrm{~b}$ & $328.1 \mathrm{~b}$ & $40.47 \mathrm{~b}$ & $39.39 \mathrm{~b}$ & $39.93 \mathrm{~b}$ & $12.03 \mathrm{~b}$ & $11.66 \mathrm{c}$ & $11.84 \mathrm{c}$ \\
\hline $90 \mathrm{~kg}$ N/fad. & $352.3 \mathrm{a}$ & $367.3 \mathrm{a}$ & $359.8 \mathrm{a}$ & $42.57 \mathrm{a}$ & $42.30 \mathrm{a}$ & $42.44 \mathrm{a}$ & $14.23 \mathrm{a}$ & $14.12 \mathrm{~b}$ & $14.17 \mathrm{~b}$ \\
\hline $120 \mathrm{~kg} \mathrm{~N} / \mathrm{fad}$. & $366.3 \mathrm{a}$ & $380.7 \mathrm{a}$ & $373.5 \mathrm{a}$ & $42.51 \mathrm{a}$ & $43.37 \mathrm{a}$ & $42.94 \mathrm{a}$ & $14.84 \mathrm{a}$ & $15.34 \mathrm{a}$ & $15.09 \mathrm{a}$ \\
\hline F-test & $* *$ & $* *$ & $* *$ & $* *$ & $* *$ & $* *$ & $* *$ & $* *$ & $* *$ \\
\hline \multicolumn{10}{|l|}{ interaction } \\
\hline $\mathbf{H} \times \mathbf{N}$ & NS & NS & $*$ & $* *$ & NS & $* *$ & $*$ & NS & $*$ \\
\hline
\end{tabular}

Table 4. Straw yield (ton/fad.), biological yield (ton/fad.) and harvest index (\%) of wheat as affected by humic acid rate, nitrogen fertilizer level and their interaction during both seasons and their combined analysis

\begin{tabular}{|c|c|c|c|c|c|c|c|c|c|}
\hline \multirow[t]{2}{*}{ Main effects and interaction } & \multicolumn{3}{|c|}{$\begin{array}{l}\text { Straw yield } \\
\text { (ton/fad.) }\end{array}$} & \multicolumn{3}{|c|}{$\begin{array}{c}\text { Biological yield } \\
\text { (ton/fad.) }\end{array}$} & \multicolumn{3}{|c|}{$\begin{array}{c}\text { Harvest index } \\
(\%)\end{array}$} \\
\hline & $\begin{array}{c}1^{\text {st }} \\
\text { season }\end{array}$ & $\begin{array}{c}2^{\text {nd }} \\
\text { season }\end{array}$ & $\overline{\text { Comb. }}$ & $\begin{array}{c}1^{\text {st }} \\
\text { season }\end{array}$ & $\begin{array}{c}2^{\text {nd }} \\
\text { season }\end{array}$ & $\overline{\text { Comb. }}$ & $\begin{array}{c}1^{\text {st }} \\
\text { season }\end{array}$ & $\begin{array}{c}2^{\text {nd }} \\
\text { season }\end{array}$ & Comb. \\
\hline \multicolumn{10}{|l|}{ Humic acid rate $(\mathrm{H})$} \\
\hline 0 kg/fad. & $2.85 \mathrm{~b}$ & $2.74 \mathrm{~b}$ & $2.80 \mathrm{~b}$ & $4.15 \mathrm{c}$ & $3.95 \mathrm{c}$ & $4.05 \mathrm{c}$ & $30.19 \mathrm{~b}$ & $29.49 \mathrm{~b}$ & $29.84 \mathrm{~b}$ \\
\hline $2 \mathrm{~kg} / \mathrm{fad}$. & $3.31 \mathrm{a}$ & $3.15 \mathrm{a}$ & $3.23 \mathrm{a}$ & $5.04 \mathrm{~b}$ & $4.83 \mathrm{~b}$ & $4.94 \mathrm{~b}$ & $34.45 \mathrm{a}$ & $34.21 \mathrm{a}$ & $34.33 \mathrm{a}$ \\
\hline 4 kg/fad. & $3.54 \mathrm{a}$ & $3.26 \mathrm{a}$ & $3.40 \mathrm{a}$ & $5.46 \mathrm{a}$ & $5.18 \mathrm{a}$ & $5.32 \mathrm{a}$ & $35.31 \mathrm{a}$ & $36.96 \mathrm{a}$ & $36.14 \mathrm{a}$ \\
\hline F-test & $*$ & $* *$ & $* *$ & $* *$ & $* *$ & $* *$ & $* *$ & $* *$ & $* *$ \\
\hline \multicolumn{10}{|l|}{ Nitrogen fertilizer level (N) } \\
\hline 0 kg N/fad. & $1.90 \mathrm{~d}$ & $1.76 \mathrm{~d}$ & $1.83 \mathrm{~d}$ & $2.87 \mathrm{~d}$ & $2.55 \mathrm{e}$ & $2.71 \mathrm{e}$ & $33.03 \mathrm{bc}$ & $30.82 \mathrm{~d}$ & $31.93 \mathrm{bc}$ \\
\hline $30 \mathrm{~kg}$ N/fad. & $2.68 \mathrm{c}$ & $2.58 \mathrm{c}$ & $2.63 \mathrm{c}$ & $3.96 \mathrm{c}$ & $3.78 \mathrm{~d}$ & $3.87 \mathrm{~d}$ & $31.81 \mathrm{c}$ & $31.40 \mathrm{~cd}$ & $31.61 \mathrm{c}$ \\
\hline $60 \mathrm{~kg}$ N/fad. & $3.54 \mathrm{~b}$ & $3.29 \mathrm{~b}$ & $3.42 \mathrm{~b}$ & $5.30 \mathrm{~b}$ & $4.99 \mathrm{c}$ & $5.15 \mathrm{c}$ & $32.71 \mathrm{c}$ & $33.59 \mathrm{bc}$ & $33.15 b$ \\
\hline $90 \mathrm{~kg} \mathrm{~N} / \mathrm{fad}$. & $3.95 \mathrm{a}$ & $3.76 \mathrm{a}$ & $3.86 \mathrm{a}$ & $6.04 \mathrm{a}$ & $5.83 \mathrm{~b}$ & $5.94 \mathrm{~b}$ & $34.40 \mathrm{ab}$ & $35.20 \mathrm{ab}$ & $34.80 \mathrm{a}$ \\
\hline $120 \mathrm{~kg} \mathrm{~N} /$ fad. & $4.09 \mathrm{a}$ & $3.86 \mathrm{a}$ & $3.98 \mathrm{a}$ & $6.27 \mathrm{a}$ & $6.11 \mathrm{a}$ & $6.19 \mathrm{a}$ & $34.64 \mathrm{a}$ & $36.77 \mathrm{a}$ & $35.71 \mathrm{a}$ \\
\hline F-test & $* *$ & $* *$ & $* *$ & $* *$ & $* *$ & $* *$ & $* *$ & $* *$ & $* *$ \\
\hline \multicolumn{10}{|l|}{ interaction } \\
\hline $\mathbf{H} \times \mathbf{N}$ & $*$ & $* *$ & $* *$ & NS & NS & $* *$ & $* *$ & $*$ & $* *$ \\
\hline
\end{tabular}

$*, * *$ and NS indicate significant at $0.05,0.01$ and not significant, respectively. 
increase due to raising humic acid application up to $4 \mathrm{Kg} / \mathrm{fad}$. It could be noticed that increasing humic acid rates from 0 to 2 and up to $4 \mathrm{Kg}$ /fad., resulted in relative increases amounted to $21.24 \%$ and $36.71 \%$ in grain yield/fad., $15.36 \%$ and $21.43 \%$ in straw yield, $21.98 \%$ and $31.36 \%$ in biological yield as well as $15.05 \%$ and $21.11 \%$ in harvest index, concerning combine analyses. The improvement of wheat grain yield and its attributes due to humic acid application may be attributed to that humic acid stimulates the biochemical processes in plants including photosynthesis and total chlorophyll content (Akinremi et al., 2000). In addition, humic acid may improve the assimilation of major and minor elements, enzyme activation and protein synthesis and increasing dry matter accumulation which consequently increased yield and quality (Ulukan, 2008). In this connection, El-Hamdi et al. (2012) showed that number of grains/spike, spike length, 1000-grain weight, grain and straw yields/fad., were significantly increased due to humic acid application.

In addition, many researchers recorded significant enhancement in grain yield and its attributes due to humic acid application (Antoun et al., 2010; Khan et al., 2010; Bakry et al., 2013; Tufail et al., 2014; Radwan et al., 2015; Anwar et al., 2016). Moreover, the obtained results are in agreement with those reported by Kandil et al. (2016) who stated that application of humic acid resulted in significant increase in number of spikes $/ \mathrm{m}^{2}$, spike length, grain weight/ spike, 1000-grain weight. Furthermore, Manal et al. (2016) showed that the highest values of yield and yield attributes of wheat were obtained by addition of humic acid as compared with the check treatment.

\section{Effect of Nitrogen Fertilizer Levels}

\section{Yield attributes}

Results presented in Tables 1, 2 and 3 reveal that all yield attributes showed positive and significant response to increasing nitrogen fertilizer levels during both growing seasons and their combined analysis. The combined results clearly showed that plant height, spike length, number and weight of grains/spike, number of spikes $/ \mathrm{m}^{2}$ and 1000-grain weight were significantly increased by raising nitrogen fertilizer levels up to $90 \mathrm{~kg} \mathrm{~N} / \mathrm{fad}$. Whereas, flag leaf area and flag leaf chlorophyll content detected positive significant response with each increase in nitrogen fertilizer level up to $120 \mathrm{~kg} \mathrm{~N} / \mathrm{fad}$.

\section{Grain, straw, biological yields and harvest index}

As shown in Table 3, straw yield and harvest index were significantly increased by raising nitrogen fertilizer levels up to $90 \mathrm{~kg} \mathrm{~N} / \mathrm{fad}$., regarding the combined results. Furthermore, grain and biological yields exhibited positive significant response due to raising nitrogen fertilizer levels up to $120 \mathrm{~kg} \mathrm{~N} /$ fad., (Table 4). Depending on the combined analysis result, the relative increase in grain yield due to $\mathrm{N}$ fertilizer application amounted to $38.33 \%, 90.66 \%$, $128.18 \%$ and $142.99 \%$ for increasing $\mathrm{N}$ fertilizer levels from 0 to $30,60,90$ and $120 \mathrm{~kg} \mathrm{~N} / \mathrm{fad}$., respectively. Also, the relative increase in biological yield due to raising $\mathrm{N}$ fertilizer levels from 0 to $30,60,90$ and $120 \mathrm{~kg} \mathrm{~N} / \mathrm{fad}$, amounted to $42.80 \%, 90.04 \%, 119.19 \%$ and $128.41 \%$, respectively.

The increment in yield attribute characters associated with raising nitrogen fertilizer levels may be ascribed to the role of nitrogen in improving wheat growth by enhancement the meristematic activity and cell division leading to increases in internodes length and number of fertile tillers and also metabolic, photosynthesis processes as well as forming filled grains consequently producing heavier grains (Seadh et al., 2009). The favorable effect of raising nitrogen fertilizer level on grain yield and its attributes may be attributed to the fact that nitrogen considers one of the major elements for plant nutrition which increases the vegetative growth of plant and compose strong plants with long spikes. In addition, nitrogen support plant to uptake other elements, leading to increasing growth of plants, consequently enhancing growth measurements and all yield components.

The compatible effect of high nitrogen fertilizer levels on increasing wheat grain yield and its attributes was also reported by Zeidan et 
al. (2005), Antoun et al. (2010), Moussa and Abdel-Maksoud (2011), Zaki et al. (2012) and Farag and El-Khawaga (2013) as well as Khaled and Hammad (2014). Moreover, the obtained results are in accordance with those stated by Kandil et al. (2016) who recorded significant increase in number of spikes $/ \mathrm{m}^{2}$, spike length, number of grains/spike, grain weight/spike, 1000 -grain weight, grain and straw yields of wheat due to raising nitrogen fertilizer level from 166,214 up to $262 \mathrm{~kg} \mathrm{~N} / \mathrm{ha}$.

\section{Effect of the Interaction}

\section{Yield attributes}

The offered results in Table 5, show the effect of the interaction between humic acid and nitrogen fertilizer levels on yield attributes. The obtained results reveal that plant height, number of spikes $/ \mathrm{m}^{2}$ and 1000-grain weight showed gradual significant positive response to raising $\mathrm{N}$ fertilizer levels up to $120 \mathrm{Kg} \mathrm{N} /$ fad., under without humic acid application. On the other hand, under the application of humic acid (2 or 4 $\mathrm{Kg} /$ fad.) the aforementioned traits were significantly and positively responded to raising $\mathrm{N}$ fertilizer levels only up to $90 \mathrm{Kg} \mathrm{N} /$ fad., In addition, weight of grains/spike showed significant increment due to raising $\mathrm{N}$ fertilizer levels up to $90 \mathrm{Kg} \mathrm{N} /$ fad., under without humic acid application and when it was applied at the highest rate $(4 \mathrm{Kg} /$ fad. $)$ while under the application of $2 \mathrm{Kg}$ humic acid/fad., weight of grains/spike showed gradual significant and positive response to raising $\mathrm{N}$ fertilizer levels up to $120 \mathrm{Kg} \mathrm{N} /$ fad. Furthermore, flag leaf area and chlorophyll content (SPAD value) presented gradual significant and positive response to increasing $\mathrm{N}$ fertilizer levels up to $120 \mathrm{Kg}$ $\mathrm{N} /$ fad., under the control treatment (without humic acid application) and the lower application of humic acid ( $2 \mathrm{Kg} / \mathrm{fad}$.), while under the application of the highest rate of humic acid (4 Kg/fad.) flag leaf area and chlorophyll content (SPAD value) were significantly increased due to raising $\mathrm{N}$ fertilizer levels up to $90 \mathrm{Kg} \mathrm{N} / \mathrm{fad}$. Weight of grains/spike exhibited significant response to raising $\mathrm{N}$ fertilizer levels up to $120 \mathrm{Kg} \mathrm{N} / \mathrm{fad}$., under the application of $2 \mathrm{Kg}$ humic acid/fad., whereas, it responded only to raising $\mathrm{N}$ fertilizer levels up to $90 \mathrm{Kg} \mathrm{N} /$ fad., under control treatment and application of $4 \mathrm{Kg}$ humic acid/fad.

It could be noticed that, the lowest values of plant height $(60.50 \mathrm{~cm})$, flag leaf area $(13.70$ $\mathrm{cm}^{2}$ ), flag leaf chlorophyll content (30.50), weight of grains/spike $(0.763 \mathrm{~g})$, number of spikes $/ \mathrm{m}^{2}$ (232) and 1000-grain weight (27.73 g) were obtained under the control treatment (without humic and without nitrogen applications). On the other direction, the highest values of plant height $(96.50 \mathrm{~cm})$ and 1000 -grain weight $(45.85 \mathrm{~g})$ could be achived under the treatment of $4 \mathrm{~kg}$ humic acid with $90 \mathrm{~kg} \mathrm{~N} /$ fad., as well as, the highest value for each of flag leaf area $\left(32.92 \mathrm{~cm}^{2}\right)$, chlorophyll content (49.02 SPAD unite) and number of spikes $/ \mathrm{m}^{2}$ (401.2) was appreciable via the concomitant of $4 \mathrm{~kg}$ humic acid with $120 \mathrm{~kg} \mathrm{~N} / \mathrm{fad}$.

\section{Grain, biological yields and harvest index}

Results presented in Table 6, clearly indicate that grain and biological yields as well as harvest index showed positive and gradual significant response to raising $\mathrm{N}$ fertilizer levels up to $120 \mathrm{Kg} \mathrm{N} /$ fad., when humic acid wasn't applied, while under the application of 2 or $4 \mathrm{Kg}$ humic acid/fad., grain and biological yields were significantly increased due to raising $\mathrm{N}$ fertilizer levels up to $90 \mathrm{Kg} \mathrm{N} / \mathrm{fad}$. However, nitrogen fertilizer levels showed no significant effect on harvest index under humic acid application (2 or $4 \mathrm{Kg} / \mathrm{fad}$. It could be noticed that, the lowest values of grain yield (4.61 ardab/fad.), biological yield (2.14 ton/fad.) and harvest index (25.27) were observed under control treatment (without humic acid + without $\mathrm{N}$ fertilizer), while the highest values of grain yield (16.25 ardab/fad.), biological yield (6.50 ton/fad.) and harvest index (37.54) were achieved under the treatment of $4 \mathrm{Kg}$ humic acid $+90 \mathrm{Kg} \mathrm{N} / \mathrm{fad}$. In this respect, Chen and Aviad (1990) reported that the stimulatory effects of humic substances have been directly related to the improved uptake of macronutrients, such as nitrogen, phosphorus and sulfur. In addition, Pettit (2004) indicated that soil application of humic substances reduced the requirement for nitrogen, phosphorus and potassium fertilizer applications. 
Table 5. Plant height $(\mathrm{cm})$, flag leaf area $\left(\mathrm{cm}^{2}\right)$, chlorophyll content (SPAD value), weight of grains/spike (g), number of spikes/ $\mathrm{m}^{2}$ and 1000 -grain weight $(\mathrm{g})$ of wheat as affected by the interaction between humic acid rates and nitrogen fertilizer levels (combined analysis)

\begin{tabular}{|c|c|c|c|c|c|}
\hline \multirow[t]{2}{*}{ Humic acid rate } & \multicolumn{5}{|c|}{ Nitrogen fertilizer level } \\
\hline & 0 kg (N/fad.) & $30 \mathrm{~kg}$ (N/fad.) & $60 \mathrm{~kg}$ (N/fad.) & $90 \mathrm{~kg}$ (N/fad.) & $120 \mathrm{~kg}$ (N/fad.) \\
\hline & \multicolumn{5}{|c|}{ Plant height (cm) } \\
\hline & $\mathrm{E}$ & $\mathrm{D}$ & $\mathrm{C}$ & $\mathrm{B}$ & A \\
\hline \multirow{2}{*}{0 kg/fad. } & $60.50 \mathrm{~b}$ & $70.33 \mathrm{~b}$ & $79.67 \mathrm{~b}$ & $84.17 \mathrm{~b}$ & $91.67 \mathrm{~b}$ \\
\hline & $\mathrm{D}$ & $\mathrm{C}$ & $\mathrm{B}$ & A & A \\
\hline \multirow{2}{*}{$2 \mathrm{~kg} / \mathrm{fad}}$. & $65.00 \mathrm{a}$ & $72.83 \mathrm{~b}$ & $84.83 \mathrm{a}$ & $92.67 \mathrm{a}$ & $95.67 \mathrm{a}$ \\
\hline & $\mathrm{D}$ & $\mathrm{C}$ & $\mathrm{B}$ & A & A \\
\hline \multirow[t]{3}{*}{$4 \mathrm{~kg} / \mathrm{fad}}$. & $67.83 \mathrm{a}$ & $78.50 \mathrm{a}$ & $85.83 \mathrm{a}$ & $96.50 \mathrm{a}$ & $94.00 \mathrm{a}$ \\
\hline & \multicolumn{5}{|c|}{ Flag leaf area $\left(\mathrm{cm}^{2}\right)$} \\
\hline & $\mathrm{E}$ & $\mathrm{D}$ & $\mathrm{C}$ & $\mathrm{B}$ & $\mathrm{A}$ \\
\hline \multirow[t]{2}{*}{0 kg/fad. } & $13.70 \mathrm{c}$ & $18.72 \mathrm{~b}$ & $23.18 \mathrm{~b}$ & $26.43 \mathrm{c}$ & $30.90 \mathrm{~b}$ \\
\hline & $\mathrm{E}$ & $\mathrm{D}$ & $\mathrm{C}$ & B & A \\
\hline \multirow[t]{2}{*}{$2 \mathrm{~kg} / \mathrm{fad}}$. & $17.05 \mathrm{~b}$ & $22.28 \mathrm{a}$ & $26.38 \mathrm{a}$ & $29.80 \mathrm{~b}$ & $32.58 \mathrm{a}$ \\
\hline & $\mathrm{D}$ & $\mathrm{C}$ & $\mathrm{B}$ & A & A \\
\hline \multirow[t]{3}{*}{$4 \mathrm{~kg} / \mathrm{fad}}$. & $20.78 \mathrm{a}$ & $23.23 \mathrm{a}$ & $27.43 \mathrm{a}$ & $32.08 \mathrm{a}$ & $32.92 \mathrm{a}$ \\
\hline & & Flag leaf chlo & rophyll content & (SPAD value) & \\
\hline & E & $\mathrm{D}$ & $\mathrm{C}$ & B & A \\
\hline \multirow{2}{*}{0 kg/fad. } & $30.50 \mathrm{c}$ & $32.30 \mathrm{c}$ & $36.47 \mathrm{c}$ & $39.32 \mathrm{c}$ & $42.03 \mathrm{~b}$ \\
\hline & $\mathrm{E}$ & $\mathrm{D}$ & $\mathrm{C}$ & $\mathrm{B}$ & A \\
\hline \multirow[t]{2}{*}{$2 \mathrm{~kg} / \mathrm{fad}}$. & $35.25 \mathrm{~b}$ & $38.10 \mathrm{~b}$ & $41.67 \mathrm{~b}$ & $43.52 \mathrm{~b}$ & $47.97 \mathrm{a}$ \\
\hline & $\mathrm{D}$ & $\mathrm{C}$ & $\mathrm{B}$ & $\mathrm{AB}$ & A \\
\hline \multirow[t]{3}{*}{4 kg/fad. } & $39.93 \mathrm{a}$ & $43.75 \mathrm{a}$ & $46.92 \mathrm{a}$ & $48.22 \mathrm{a}$ & $49.02 \mathrm{a}$ \\
\hline & \multicolumn{5}{|c|}{ Weight of grains/spike (g) } \\
\hline & D & C & B & A & A \\
\hline \multirow[t]{2}{*}{0 kg/fad. } & $0.763 \mathrm{~b}$ & $0.933 \mathrm{c}$ & $1.107 \mathrm{c}$ & $1.393 \mathrm{c}$ & $1.487 \mathrm{~b}$ \\
\hline & E & $\mathrm{D}$ & $\mathrm{C}$ & B & A \\
\hline \multirow[t]{2}{*}{$2 \mathrm{~kg} / \mathrm{fad}}$. & $0.947 \mathrm{a}$ & $1.203 \mathrm{~b}$ & $1.470 \mathrm{~b}$ & $1.762 \mathrm{~b}$ & $1.885 \mathrm{a}$ \\
\hline & $\mathrm{D}$ & $\mathrm{C}$ & B & A & A \\
\hline \multirow[t]{3}{*}{4 kg/fad. } & \multirow{2}{*}{\multicolumn{5}{|c|}{ Number of spikes $/ \mathrm{m}^{2}$}} \\
\hline & & & & & \\
\hline & E & D & C & B & A \\
\hline \multirow[t]{2}{*}{0 kg/fad. } & $232.0 \mathrm{~b}$ & $254.5 \mathrm{~b}$ & $278.7 \mathrm{~b}$ & $308.5 \mathrm{~b}$ & $339.8 \mathrm{~b}$ \\
\hline & $\mathrm{D}$ & $\mathrm{C}$ & $\mathrm{B}$ & A & A \\
\hline \multirow[t]{2}{*}{$2 \mathrm{~kg} / \mathrm{fad}}$. & $281.2 \mathrm{a}$ & $319.0 \mathrm{a}$ & $340.2 \mathrm{a}$ & $376.7 \mathrm{a}$ & $379.5 \mathrm{a}$ \\
\hline & $\mathrm{D}$ & $\mathrm{C}$ & B & A & A \\
\hline \multirow[t]{3}{*}{$4 \mathrm{~kg} / \mathrm{fad}}$. & $281.8 \mathrm{a}$ & $313.8 \mathrm{a}$ & $365.3 \mathrm{a}$ & $394.3 \mathrm{a}$ & $401.2 \mathrm{a}$ \\
\hline & \multicolumn{5}{|c|}{1000 -grain weight (g) } \\
\hline & $\mathrm{E}$ & $\mathrm{D}$ & $\mathrm{C}$ & B & $\mathrm{A}$ \\
\hline \multirow[t]{2}{*}{0 kg/fad. } & $27.73 \mathrm{c}$ & $32.17 \mathrm{c}$ & $36.62 \mathrm{c}$ & $39.55 \mathrm{c}$ & $41.83 \mathrm{~b}$ \\
\hline & $\mathrm{D}$ & $\mathrm{C}$ & B & A & A \\
\hline \multirow{2}{*}{$2 \mathrm{~kg} / \mathrm{fad}$} & $33.27 \mathrm{~b}$ & $37.25 \mathrm{~b}$ & $40.32 \mathrm{~b}$ & $42.05 \mathrm{~b}$ & $42.45 \mathrm{~b}$ \\
\hline & $\mathrm{D}$ & $\mathrm{C}$ & B & A & A \\
\hline $4 \mathrm{~kg} / \mathrm{fad}$. & $35.62 \mathrm{a}$ & $39.80 \mathrm{a}$ & $42.85 \mathrm{a}$ & $45.70 \mathrm{a}$ & $44.53 \mathrm{a}$ \\
\hline
\end{tabular}


Table 6. Grain yield (ardab/fad.), biological yield (ton/fad.) and harvest index (\%)of wheat as affected by the interaction between humic acid rates and nitrogen fertilizer levels (combined analysis)

\begin{tabular}{|c|c|c|c|c|c|}
\hline \multirow[t]{2}{*}{$\overline{\text { Humic acid rate }}$} & \multicolumn{5}{|c|}{ Nitrogen fertilizer level } \\
\hline & $0 \mathrm{~kg} \mathrm{~N} /$ fad. & $30 \mathrm{~kg} \mathrm{~N} / \mathrm{fad}$ & $60 \mathrm{~kg} \mathrm{~N} / \mathrm{fac}$ & $90 \mathrm{~kg} \mathrm{~N} / \mathrm{fad}$ & $20 \mathrm{~kg} \mathrm{~N} /$ fad. \\
\hline & \multicolumn{5}{|c|}{ Grain yield (ardab/fad.) } \\
\hline & $\mathrm{E}$ & $\mathrm{D}$ & $\mathrm{C}$ & $\mathrm{B}$ & A \\
\hline \multirow[t]{2}{*}{0 kg/fad. } & $4.61 \mathrm{c}$ & $6.43 \mathrm{~b}$ & $9.49 \mathrm{c}$ & $12.14 \mathrm{c}$ & $14.17 \mathrm{c}$ \\
\hline & $\mathrm{D}$ & $\mathrm{C}$ & $\mathrm{B}$ & $\mathrm{A}$ & $\mathrm{A}$ \\
\hline \multirow[t]{2}{*}{$2 \mathrm{~kg} / \mathrm{fad}$. } & $6.38 \mathrm{~b}$ & $9.20 \mathrm{a}$ & $12.14 \mathrm{~b}$ & $14.13 \mathrm{~b}$ & $14.96 \mathrm{~b}$ \\
\hline & $\mathrm{D}$ & $\mathrm{C}$ & B & A & A \\
\hline \multirow[t]{3}{*}{4 kg/fad. } & $7.62 \mathrm{a}$ & $10.14 \mathrm{a}$ & $13.90 \mathrm{a}$ & $16.25 \mathrm{a}$ & $16.15 \mathrm{a}$ \\
\hline & \multicolumn{5}{|c|}{ Biological yield (ton/fad.) } \\
\hline & $\mathrm{E}$ & $\mathrm{D}$ & $\mathrm{C}$ & $\mathrm{B}$ & $\mathrm{A}$ \\
\hline \multirow[t]{2}{*}{0 kg/fad. } & $2.14 \mathrm{~b}$ & $2.97 \mathrm{c}$ & $4.28 \mathrm{c}$ & $5.19 \mathrm{c}$ & $5.66 \mathrm{~b}$ \\
\hline & $\mathrm{D}$ & $\mathrm{C}$ & $\mathrm{B}$ & $\mathrm{A}$ & $\mathrm{A}$ \\
\hline \multirow[t]{2}{*}{$2 \mathrm{~kg} / \mathrm{fad}$. } & $2.87 \mathrm{a}$ & $3.97 \mathrm{~b}$ & $5.40 \mathrm{~b}$ & $6.10 \mathrm{~b}$ & $6.35 \mathrm{a}$ \\
\hline & $\mathrm{D}$ & $\mathrm{C}$ & $\mathrm{B}$ & $\mathrm{A}$ & A \\
\hline \multirow[t]{3}{*}{4 kg/fad. } & $3.11 \mathrm{a}$ & $4.66 \mathrm{a}$ & $5.75 \mathrm{a}$ & $6.50 \mathrm{a}$ & $6.57 \mathrm{a}$ \\
\hline & \multicolumn{5}{|c|}{ Harvest index (\%) } \\
\hline & $\mathrm{D}$ & $\mathrm{CD}$ & $\mathrm{C}$ & $\mathrm{B}$ & A \\
\hline \multirow[t]{2}{*}{$0 \mathrm{~kg} / \mathrm{fad}$. } & $25.27 \mathrm{c}$ & $27.49 \mathrm{~b}$ & $29.54 \mathrm{c}$ & $32.07 \mathrm{c}$ & 34.84 \\
\hline & A & $\mathrm{A}$ & A & $\mathrm{A}$ & $\mathrm{A}$ \\
\hline \multirow[t]{2}{*}{$2 \mathrm{~kg} / \mathrm{fad}$. } & $33.14 \mathrm{~b}$ & $34.70 \mathrm{a}$ & $33.64 \mathrm{~b}$ & $34.79 \mathrm{~b}$ & 35.38 \\
\hline & A & $\mathrm{B}$ & A & A & A \\
\hline $4 \mathrm{~kg} / \mathrm{fad}$. & $37.37 \mathrm{a}$ & $32.63 \mathrm{a}$ & $36.27 \mathrm{a}$ & $37.54 \mathrm{a}$ & 36.88 \\
\hline
\end{tabular}

\section{REFERENCES}

Akinremi, O.O., H.H. Janzen, R.L. Lemke and F.J. Larney (2000). Response of canola, wheat and green beans to leonardite additions. Canadian J. Soil Sci., 80: 437-443.

Ali, A.G.A., O.E. Zeiton, A.H. Bassiauny and A.R.Y.A. El-Banna (2004). Productivity of wheat cultivars grown at El-Khattra and ElArish under different levels of planting densities and $\mathrm{N}$-fertilization. Zagazig J. Agric. Res., 31 (4A): 1225-1256.

Antoun, L.W., S.M. Zakaria and H.H. Rafla (2010). Influence of compost, N-mineral and humic acid on yield and chmical composition of wheat plants. J. Soil Sci. and Agric. Eng., Mansoura Univ., 1 (11): 1131- 1143.
Anwar, S., F. Iqbal, W. A. Khattak, M. Islam, B. Iqbal and S. Khan (2016). Response of wheat crop to humic acid and nitrogen levels. EC Agric., 3 (1): 558-565.

Asik, B.A., M.A. Turan., H. Celik and A.V. Katkat. (2009). Effects of humic substances on plant growth and mineral nutrients uptake of wheat (Triticum durum cv. Salihli) under conditions of salinity. Asian J. Crop Sci., 1 (2): 87-95.

Bakry, B.A., T.A. Elewa., M.F. El-Kramany and A.M. Wali (2013). Effect of humic and ascorbic acids foliar application on yield and yield components of two wheat cultivars grown under newly reclaimed sandy soil. Int. J. Agron. Plant. Prod., 4 (6): 1125- 1133. 
Castelli, F., R. Contillo and F. Miceli (1996). Non-destructive determination of leaf chlorophyll content in four crop species. J. Agron. and Crop Sci., 177: 275-283.

Chen, Y. and T. Aviad (1990). Effects of humic substance on plant growth. In MacCarthy, CE Clapp, RL Malcolm, PR Bloom (eds.). Humic substances in soil and crop sciences: Selected readings. Soil Sci. Soc. Ame., Madison. Wisconsin, 161-186.

Chen, Y., Clapp, C.H. El-Magen and V.W. Cline (1999). Stimulation of plant growth by humic substances: Effects on iron availability. In: Ghabbour EA, Davies G (eds.). Understanding humic substances: Advanced methods, properties and applications. R. Soc. Chem. Cambridge. UK, 255-263.

Cimrin, K.M. and I. Yilmaz (2005). Humic acid applications to lettuce do not improve yield but do improve phosphorus availability. Acta Agric. Scandinavica, Section B, Soil and Plant Sci., 55: 58-63.

El-Hamdi, Kh. H., E. M. Selim and H. I. M. Husein (2012). Integrated impacts of humic acid, halotolerant $\mathrm{N}_{2}$ fixers and nitrogen application on wheat yied (Triticum aestivum L.), yield component and nutrient uptake. J. Soil Sci. and Agric. Eng., Mansoura Univ., 3 (12): 1263 - 1274 .

FAOSTAT (2016). Food and Agricultural Organization of the United Nations (FAO), FAO Statistical Database, from http:// faostat. fao.org. December 2015.

Farag, I.A.A. and A.A.H. El-Khawaga (2013). Influnce of gamma irradiation and nitrogen fertilizer levels on Gemmeza-9 wheat cultivar yield and its attridutes. Arab J. Nuclear Sci. and Applic., 46 (2): 363-371.

Gomez, K.A. and A.A. Gomez (1991). Statistical Procedures for Agricultural Research. $2^{\text {nd }}$ Ed., Jhon Wiley and Sons Inc., New York, 95-109.

Kandil, A.A., A.E.M. Sharief, S.E. Seadh and D.S.K. Altai (2016). Role of humic acid and amino acids in limiting loss of nitrogen fertilizer and increasing productivity of some wheat cultivars grown under newly reclaimed sandy soil. Int. J. Adv. Res. Biol. Sci., 3(4): 123-136.

Khaled, H. and H.A. Fawy (2011). Effect of different levels of humic acids on the nutrient content, plant growth, and soil properties under conditions of salinity. Soil and Water Res., 6 (1): 21-29.

Khaled, M.A. and S.M. Hammad (2014). Effect of nitrogen and potassium levels on yield and its components of four new bread wheat cultivars. J. Plant Prod., Mansoura Univ., 5 (1): 95- 105.

Khan, R.U., A. Rashid, M.S. Khan and E. Ozturk (2010). Impact of humic acid and chemical fertilizer application on growth and grain yield of rainfed wheat (Triticum aestivum L.). Pak. J. Agric. Res., 23 (3-4): 113-121.

Lui, K.N. and M.S. Subbo Roo (1951). A rapid method of leaf area determination. Nat., 167172.

Manal, F.M., A.T. Thalooth, A.G. Ahmed, M.H. Mohamed and T.A. Elewa (2016). Evaluation of the effect of chemical fertilizer and humic acid on yield and yield components of wheat plants (Triticum aestivum, L.) grown under newly reclaimed sandy soil. Int. J. Chem. Tech. Res., 9 (8): 154-161.

Moussa, A.M. and H.H. Abdel-Maksoud (2011). Response of grian yield and some its components, water productivity and nitrogen use efficiency for some bread wheat cultivars to $\mathrm{N}$-rate under deficit irrigation, induced via corrugation metod, on vertisol. Alex. J. Agric. Res., 56 (1): 27-38.

Mowafy, S.A.E. (2008). Effect of organic manure and $\mathrm{N}$ fertilization level on floral fertility, inter and intra spikelet competition and grain yield potentiality of three bread wheat cultivars under sandy soil conditions. Zagazig J. Agric. Res., 35 (5): 1015-1052.

MSTAT-C. (1991). A Microcomputer program for the design, management and analysis of agronomic research experiment. MSTAT Develop. Team, Michigan State Univ.

Nardi, S., D. Pizeghello, A. Muscdo and A. Vianello (2002). Physiological effects of humic substances on higher plants. Soil Biochem., 34: 1527- 1536. 
Pettit, R.E. (2004). Organic matter, humus, humate, humic acid, fulvic acid and humin: Their importance in soil fertility and plant health [Online]. Available at www.humate. info/mainpage.htm.

Radwan, F.I., M.A. Gomaa, I.F. Rehab and S.I.A. Adam (2015). Impact of humic acid application, foliar micronutrients and biofertilization on growth, productivity and quality of wheat (Triticum aestivum, L.). Middle East J. Agric. Res., 4 (2): 130-140.

Seadh S.E., M.I. El-Abady, A.M. El-Ghamry and S. Farouk (2009). Influence of micronutrients foliar application and nitrogen fertilization on wheat yield and quality of grain and seed. J. Biol. Sci., 9 (8): 851-858.

Steel, R.G.D., J.H. Torrie and D.H. Dickey (1997). Principles and Procedures of Statistics. A. Biometrical Approach, $3^{\text {rd }}$ Ed. Mc Graw Hill. Book Co. New Yourk, USA, 352-358
Tufail, M., K. Nawaz and M. Usman (2014). Impact of humic acid on the morphology and yeild of wheat (Triticum aestivum L.). World Appl. Sci. J., 30 (4): 475-480.

Ulukan, H. (2008). Effect of soil applied humic acid at different sowing times on some yield components in wheat (Triticum spp.) hybrids Int. J. Bot., 4(2): 164-175.

Zaki, N.M., M.A. Gomaa, F.I. Radwan, M.S. Hassanein and A.M. Wali (2012). Effect of mineral, organic and Bio- Fertilizers on yield, yield components and chemical composition of some wheat cultivars. J. Appl. Sci. Res., 8 (1): 174-191.

Zeidan, E.M., A.A. El-Khawaga, H.A. Basha and I.M. Abd El-Hammeed (2005). Improvement of wheat productivity in newly reclaimed soil in Egypt. Annals UMCS, 60: $113-121$.

\section{تأثير إضافة حامض الهيوميك ومستوى السماد النيتروجيني على إنتاجية قمح الخبز تحت ظروف الأراضي الرملية

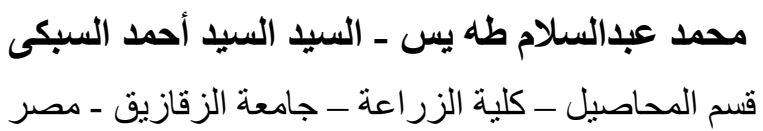

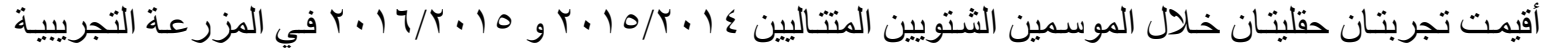

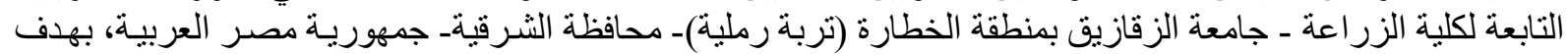

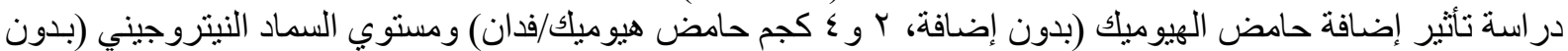

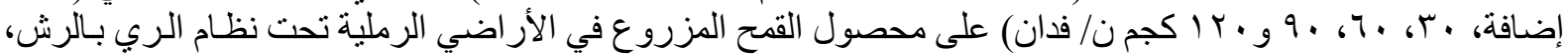

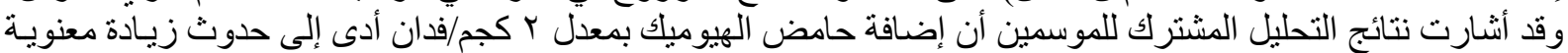

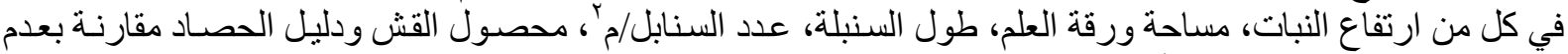

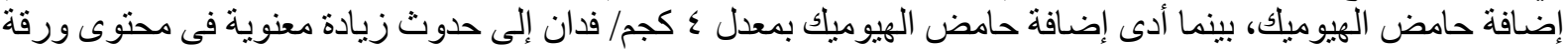

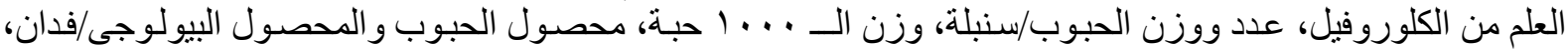

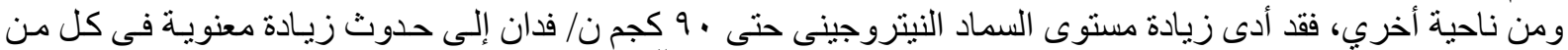

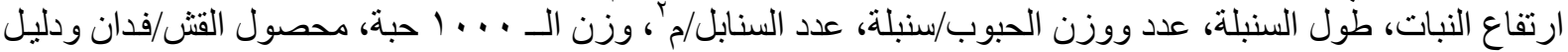

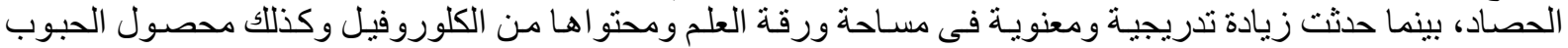

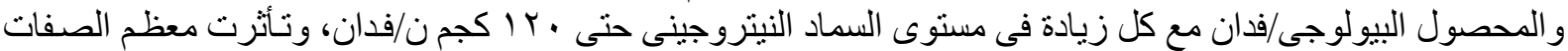

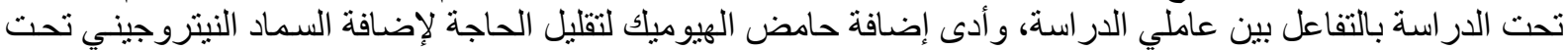

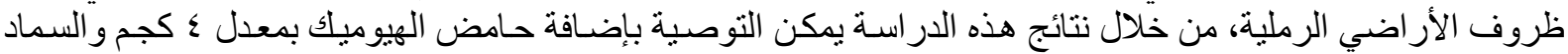

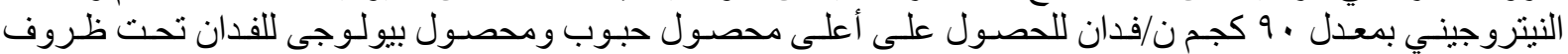
الأر اضي الرملية بمحافظة الثرقية. 